\title{
Supplementation with psyllium seed husk reduces myocardial damage in a rat model of ischemia/reperfusion
}

\author{
Sun Ha Lim and Jongwon Lee ${ }^{\S}$ \\ Department of Biochemistry, School of Medicine, Catholic University of Daegu, 33 Duryugongwon-ro 17-gil, Nam-gu, Daegu 42472, Republic of Korea
}

BACKGROUND/OBJECTIVES: Myocardial infarction (MI) is caused by extensive myocardial damage attributed to the occlusion of coronary arteries. Our previous study in a rat model of ischemia/reperfusion (I/R) demonstrated that administration of arabinoxylan (AX), comprising arabinose and xylose, protects against myocardial injury. In this study, we undertook to investigate whether psyllium seed husk (PSH), a safe dietary fiber containing a high level of AX (>50\%), also imparts protection against myocardial injury in the same rat model.

MATERIALS/METHODS: Rats were fed diets supplemented with PSH (1, 10, or $100 \mathrm{mg} / \mathrm{kg} / \mathrm{d})$ for $3 \mathrm{~d}$. The rats were then subjected to $30 \mathrm{~min}$ ischemia through ligation of the left anterior descending coronary artery, followed by $3 \mathrm{~h}$ reperfusion through release of the ligation. The hearts were harvested and cut into four slices. To assess infarct size (IS), an index representing heart damage, the slices were stained with 2,3,5-triphenyltetrazolium chloride (TTC). To elucidate underlying mechanisms, Western blotting was performed for the slices.

RESULTS: Supplementation with 10 or $100 \mathrm{mg} / \mathrm{kg} / \mathrm{d}$ of PSH significantly reduces the IS. PSH supplementation (100 mg/kg/d) tends to reduce caspase-3 generation and increase BCL-2/BAX ratio. PSH supplementation also upregulates the expression of nuclear factor erythroid 2-related factor 2 (NRF2), and its target genes including antioxidant enzymes such as glutathione S-transferase mu 2 (GSTM2) and superoxide dismutase 2 (SOD2). PSH supplementation upregulates some sirtuins (NAD ${ }^{+}$-dependent deacetylases) including SIRT5 (a mitochondrial sirtuin) and SIRT6 and SIRT7 (nuclear sirtuins). Finally, PSH supplementation upregulates the expression of protein kinase $A(P K A)$, and increases phosphorylated cAMP response element-binding protein (CREB) (pCREB), a target protein of PKA.

CONCLUSIONS: The results from this study indicate that PSH consumption reduces myocardial I/R injury in rats by inhibiting the apoptotic cascades through modulation of gene expression of several genes located upstream of apoptosis. Therefore, we believe that PSH can be developed as a functional food that would be beneficial in the prevention of MI.

Nutrition Research and Practice 2019;13(3):205-213; https://doi.org/10.4162/nrp.2019.13.3.205; pISSN 1976-1457 elSSN 2005-6168

Keywords: Infarction, apoptosis, sirtuin, functional food

\section{INTRODUCTION}

A subset of coronary heart disease (CHD), myocardial infarction (MI) is a consequence of irreversible heart damage. The damage results from the extensive death of myocardial cells, and is attributed to prolonged ischemia with or without reperfusion, generated by occlusion of the coronary arteries. Progression to $\mathrm{Ml}$ can be divided into the preocclusion and postocclusion steps [1-4] (Fig. 1).

In the preocclusion steps, atherosclerosis results in narrowing of the arteries, which, in turn, is triggered by major risk factors such as hypertension, hypercholesterolemia, and diabetes [5, 6]. These risk factors exert oxidative stress on the endothelium resulting in endothelial dysfunction, which permits low-density lipoprotein (LDL) to enter the intima [7-9]. The LDL absorbed is oxidized and then engulfed by macrophages, which transform into foam cells. Proliferation of the foam cells leads to plaque formation, resulting in narrowing of the arteries. Overall, this is a slow process and usually takes several decades [10]. Previous studies have demonstrated the efficacy of psyllium seed husk (PSH) consumption in reducing risk factors such as hyperlipidemia [11-13], diabetes [14], and hypertension [15,16], thereby inhibiting the preocclusion steps (Fig. 1).

In the postocclusion steps, abrupt rupture of the plaques results in clot formation which, in turn, occludes the artery and results in myocardial ischemia. The resultant ischemia greatly

\footnotetext{
This work was supported by a grant of Comprehensive and Integrative Medicine R\&D project through Comprehensive and Integrative Medicine Institute (CIMI), funded by the Ministry of Health \& Welfare (MOHW), Republic of Korea (Grant Number : CIMI-15-02-08), the Basic Science Research Program through the National Research Foundation of Korea (NRF) funded by the Ministry of Education (No. 2017R1D1A1B03034255) to J. L., and supported by the National Research Foundation of Korea (NRF) grant funded by the Korea government(MSIT) (No. NRF-2017R1C1B2010220) to S. H. L.

${ }^{\S}$ Corresponding Author: Jongwon Lee, Tel. 82-53-650-4471, Fax. 82-53-621-4106, Email. leejw@cu.ac.kr Received: December 17, 2018, Revised: March 13, 2019, Accepted: March 23, 2019

This is an Open Access article distributed under the terms of the Creative Commons Attribution Non-Commercial License (http://creativecommons.org/licenses/by-nc/3.0/) which permits unrestricted non-commercial use, distribution, and reproduction in any medium, provided the original work is properly cited.
} 


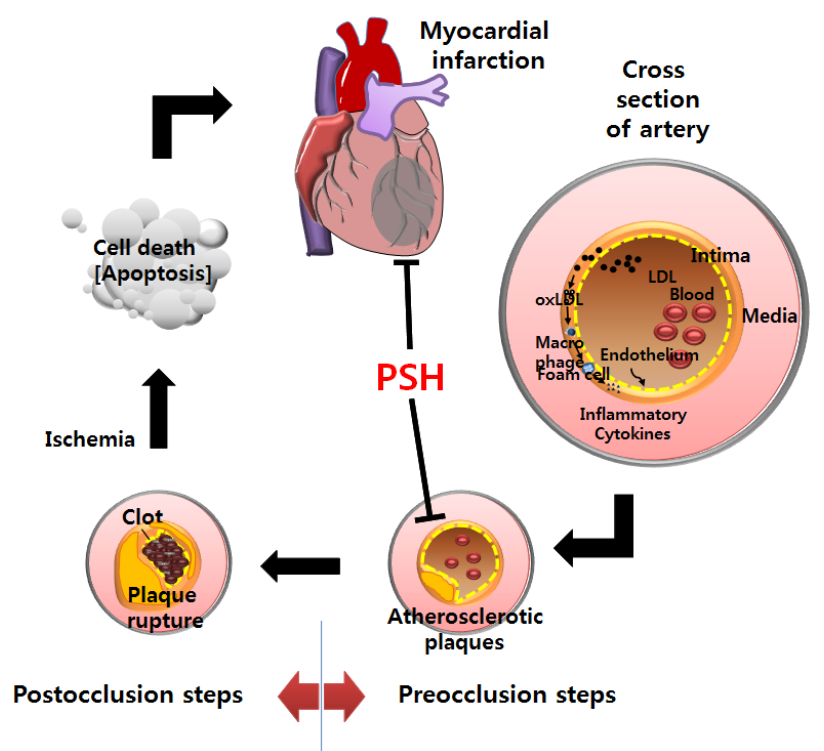

Fig. 1. Summary of development of myocardial infarction (Modified from [4]). In the preocclusion steps, LDL enters the intima due to endothelial dysfunction. LDL absorbed is oxidized to oxLDL, and oxLDL is engulfed by the macrophages. The macrophages are then transformed to foam cells which subsequently proliferate, resulting in the formation of atherosclerotic plaques and narrowing of the arteries. In the post-occlusion steps, abrupt rupture of the plaques leads to clot formation in the lesion. This event can occlude the artery and subsequently result in myocardial ischemia. As a result, ATP generation is greatly reduced due to interruption of oxidative phosphorylation, and myocardial cells die through apoptosis and necrosis. As regions of cell death become extensive, myocardial infarction ensues. PSH, psyllium seed husk; LDL, low-density lipoprotein; oxLDL, oxidized LDL.

reduces ATP generation and produces excessive reactive oxygen species (ROS) from the cytosol and mitochondria when coupled with reperfusion, in particular. Consequently, myocardial cells get damaged and die through necrosis and apoptosis, including generation of caspase 3 from procaspase 3, subsequently leading to Ml. ROS, including superoxide anion $\left(\mathrm{O}_{2}\right)$ and hydrogen peroxide $\left(\mathrm{H}_{2} \mathrm{O}_{2}\right)$, is mainly generated in the mitochondria [17]. ROS reacts with polyunsaturated fatty acids (PUFA) such as cardiolipin (a mitochondrial lipid), resulting in the production of toxic byproducts such as 4-hydroxy-2-nonenal (HNE) and malondialdehyde (MDA) [17]. ROS levels are reduced by the transformation of $\mathrm{O}_{2}^{-}$to $\mathrm{H}_{2} \mathrm{O}_{2}$ by superoxide dismutase (SOD) [18], followed by the conversion of $\mathrm{H}_{2} \mathrm{O}_{2}$ into innocuous water by a catalase reaction [18]. On the other hand, 4-hydroxynoneal (HNE) is removed by glutathione S-transferase (GST) through conjugation with glutathione [19]. Antioxidant enzymes, including SOD, catalase, and GST, are target genes of the transcription factor nuclear factor erythroid 2-related factor 2 (NRF2), which attach to the binding sites on promoters of the target genes, thereby upregulating transcription of the antioxidant enzymes $[20,21]$. In addition, several proteins located upstream of NRF2 in the apoptotic cascades such as sirtuins [22], cAMP response element-binding protein (CREB) [23], and protein kinase A (PKA) [24], are involved in modulating myocardial I/R injury. This is a fast process and usually takes only several days for humans [25].

In our previous study, we demonstrated that administration of arabinoxylan (AX; a major cell wall polysaccharide of wheat consisting of arabinose and xylose), arabinose itself, as well as hot water extract of ground wheat, reduces myocardial injury at the postocclusion steps in a rat MI model [26]. Our findings implicate that any foods containing high levels of $A X$ may be efficacious in preventing $\mathrm{Ml}$ through the action of arabinose during the postocclusion steps. To test the hypothesis, we conducted a literature search and chose PSH (soluble dietary fiber) as a candidate since PSH is rich in AX (approximately 60 wt $\%$ of PSH) and yields approximately 20 and 46 wt $\%$ of arabinose and xylose, respectively, when hydrolyzed [27]. Based on these findings, we undertook to investigate whether PSH supplementation for $3 \mathrm{~d}$ reduces the myocardial injury in a rat model of $\mathrm{Ml}$ where the left anterior descending coronary artery (LAD) was occluded for $30 \mathrm{~min}$ through ligation, and then reperfused for $3 \mathrm{~h}$ through release of ligation. We further investigated whether PSH supplementation reduces the myocardial injury by inhibiting the apoptotic cascades, including PKA to caspase-3 (CASP3). This study provides some evidence indicating that PSH is able to prevent Ml by inhibiting only the postocclusion steps, since the $3 \mathrm{~d}$ period chosen to supplement PSH is too short to affect the preocclusion steps which usually takes several decades [10] (Fig. 1).

\section{MATERIALS AND METHODS}

\section{Materials}

PSH was purchased from Pharmatech Korea (Yongin, Gyeonggido, Republic of Korea). Other reagents were purchased from Sigma-Aldrich (St. Louis, MO, USA), unless stated otherwise. PSH was analyzed to contain approximately $66 \%$ AX and $21 \%$ arabinose (wt/wt) [28].

\section{Animals}

Eight-week-old male Sprague Dawley (SD) rats were purchased from Samtaco Inc. (Osan, Gyeonggi-do, Republic of Korea). Experiments were carried out according to the protocols for animal care and use of laboratory animals, approved by the Institutional Animal Care and Research Advisory Committee of Catholic University, Daegu, South Korea (No. DCIAFCR-15123020-Y). Animals were housed with food and water available ad libitum under diurnal lighting conditions and in a temperaturecontrolled environment until the start of the experiment.

\section{Diet Preparation}

A diet containing PSH was prepared as previously described $[1,29]$. To prepare $1 \mathrm{~kg}$ each of the 1,10 , or $100 \mathrm{mg} / \mathrm{kg} \mathrm{PSH}$ diets, mixtures of $0.02 \mathrm{~g} \mathrm{PSH}+49.98 \mathrm{~g}$ corn starch, $0.2 \mathrm{~g}$ PSH $+49.8 \mathrm{~g}$ corn starch, and $2 \mathrm{~g} \mathrm{PSH}+48 \mathrm{~g}$ corn starch were added, respectively, to $950 \mathrm{~g}$ of a modified AIN-93G diet purchased from Unifaith Inc. (Seoul, Republic of Korea) (Table 1). To prepare $1 \mathrm{~kg}$ of the basal diet, $50 \mathrm{~g}$ of corn starch was added to $950 \mathrm{~g}$ of the modified AIN-93G diet.

\section{Diet administration}

PSH diets and basal diet were supplied to the rats as described previously $[1,29]$. Briefly, the rats were randomly assigned to one of the five groups: (1) sham $(n=6),(2)$ control $(\mathrm{n}=6)$, and (3) PSH-treated group (1, 10, or $100 \mathrm{mg} / \mathrm{kg}$ per day) 
Table 1. Ingredient composition of experimental diets

\begin{tabular}{|c|c|c|c|c|c|}
\hline Ingredient & $\begin{array}{c}\text { Modified } \\
\text { AIN-93 diet }(\mathrm{g} / 950 \mathrm{~g})^{1)}\end{array}$ & Basal diet $(\mathrm{g} / \mathrm{kg})^{2)}$ & $\begin{array}{c}1 \mathrm{mg} / \mathrm{kg} / \mathrm{d} \\
\text { PSH diet }(\mathrm{g} / \mathrm{kg})^{3)}\end{array}$ & $\begin{array}{c}10 \mathrm{mg} / \mathrm{kg} / \mathrm{d} \\
\text { PSH diet }(\mathrm{g} / \mathrm{kg})^{3)}\end{array}$ & $\begin{array}{c}100 \mathrm{mg} / \mathrm{kg} / \mathrm{d} \\
\text { PSH diet }(\mathrm{g} / \mathrm{kg})^{3)}\end{array}$ \\
\hline Casein & 250.0 & 250.0 & 250.0 & 250.0 & 250.0 \\
\hline Corn starch & 482.5 & 482.5 & 482.5 & 482.5 & 482.5 \\
\hline Sucrose & 100.0 & 100.0 & 100.0 & 100.0 & 100.0 \\
\hline Soybean oil & 70.0 & 70.0 & 70.0 & 70.0 & 70.0 \\
\hline Mineral mix & 35.0 & 35.0 & 35.0 & 35.0 & 35.0 \\
\hline Vitamin mix & 10.0 & 10.0 & 10.0 & 10.0 & 10.0 \\
\hline Choline bitartrate & 2.5 & 2.5 & 2.5 & 2.5 & 2.5 \\
\hline Corn starch & 0.0 & 50.0 & 49.98 & 49.8 & 48.0 \\
\hline $\mathrm{PSH}^{4)}$ & 0.0 & 0.0 & 0.02 & 0.2 & 2.0 \\
\hline t-Butylhydroquinone & 0.014 & 0.014 & 0.014 & 0.014 & 0.014 \\
\hline
\end{tabular}

1) Modified AIN-93G diet was purchased in a pre-mix form.

2) One $\mathrm{kg}$ of basal diet was prepared by adding $50 \mathrm{~g}$ of corn starch to $950 \mathrm{~g}$ of the modified AIN-93G diet.

3) One kg of PSH diets was prepared by adding $50 \mathrm{~g}$ of mixture of corn starch and psyllium seed husk (PSH) to $950 \mathrm{~g}$ of the modified AlN-93G diet.

4) $\mathrm{PSH}$ diets of 1,10 or $100 \mathrm{mg} / \mathrm{kg} / \mathrm{d}$ refer to the corresponding dosage of $\mathrm{PSH}$, given per kilogram of rat per day.

( $n=6$ per group). In the PSH-treated group, the rats (approximately $300 \mathrm{~g}$ weight) received $15 \mathrm{~g} / \mathrm{d}$ of PSH diet $(1,10$, or $100 \mathrm{mg} / \mathrm{kg} / \mathrm{d}$ PSH per rat) for $3 \mathrm{~d}$ before ligation. Once the rats consumed the requisite PSH diet each day, more basal diet was provided ad libitum. Rats in the control and sham groups received the basal diet only.

\section{Myocardial infarction model}

Ischemia/reperfusion injury was generated through ligation of the LAD followed by release of the ligation, as described previously $[1,29]$. Briefly, the male SD rats $(\sim 300 \mathrm{~g})$ were anesthetized through intramuscular injections of ketamine (100 $\mathrm{mg} / \mathrm{kg}$ ) and xylazine ( $5 \mathrm{mg} / \mathrm{kg}$ ), intubated, and ventilated with air throughout the experiment. The heart was then exposed by a left thoracic incision, and the LAD of the rats in the PSH-treated group was ligated for $30 \mathrm{~min}$ approximately $5 \mathrm{~mm}$ below the aortic origin, by passing a 5-0 Prolene suture (BV-1, Ethicon, Somerville, NJ, USA) and double-knotting the suture. Occlusion generated through the ligation was confirmed by observing development of a pale color in the left ventricular wall. Subsequently, the heart was reperfused for $3 \mathrm{~h}$ by releasing the ligation. Rats in the sham group underwent the same experimental procedure, but without ligation. During surgery, the rectal temperature was maintained at $37 \pm 0.5^{\circ} \mathrm{C}$ using a thermostat-controlled warming plate (Harvard Apparatus, Holliston, MA, USA).

\section{Assessment of the infarct size}

The infarct size (IS) was assessed through 2,3,5-triphenyltetrazolium chloride (TTC) staining, as described previously [1, 29]. After the I/R procedure, the LAD was re-ligated, and $1 \mathrm{ml}$ of $1 \%$ Evans blue dye (Sigma-Aldrich, St. Louis, MO, USA) was infused into the heart through the jugular vein. Next, the rats were euthanized by thoracotomy under anesthetization. The heart was harvested, excised into 4 pieces approximately 3-mm thick, which were subsequently stained with TTC. The area at risk (AAR) is defined as the area not infiltrated by Evans blue dye. The infarct area (IA) is defined as the area not stained with TTC. The border zone area (BZA) is defined as the area in which the IA is excluded from the AAR (AAR-IA). The AAR, IA, BZA, and left ventricular area (LVA) were determined by computerized planimetry using ImageJ software ( $\mathrm{NIH}, \mathrm{v} 1.47)$. Using these areas, we calculated the IS and risk size (RS), which are defined as the percentage of IA to AAR and AAR to LVA, respectively.

\section{Western blotting}

Western blotting was performed for the pieces of the hearts harvested, as described previously [1]. Briefly, the harvested pieces were lysed with radioimmunoprecipitation assay (RIPA) buffer (Cell Signaling, Beverly, MA, USA) containing protease inhibitor cocktail. Equal quantity of protein extracts in the supernatant were separated on sodium dodecyl sulfate (SDS)polyacrylamide gels, transferred to polyvinylidene fluoride (PVDF) membranes (Bio-Rad Laboratories, Inc., Hercules, CA, USA), and blocked with $5 \%$ skim milk, prior to incubation with each primary antibody. The primary antibodies used were cleaved CASP3 (C-CASP3, also expressed as CASP3), BCL-2, BAX, CREB, phosphorylated CREB (pCREB), superoxide dismutase 2 (SOD2), sirturin 1(SIRT1), SIRT2, SIRT3, SIRT5, SIRT6, and SIRT7 (1:1000, Cell Signaling, Beverly, MA, USA), PKA $\beta$ and SIRT4 (1:1000, Abcam, Cambridge, MA, USA), NRF2 (1:1000, Enzo, Farmingdale, NY, USA), glutathione S-transferase mu 2(GSTM2) (1:400, USCN Life Science Inc., Houston, Texas, USA), and ERK1 (1:1000, Santa Cruz Biotechnology Inc., Santa Cruz, CA, USA). The probed membranes were then incubated with the horseradish peroxidase-labeled secondary antibodies (1:2000, Enzo, Farmingdale, NY, USA) and subsequently developed with enhanced chemiluminescence $(E C L)$ substrate solution (Thermo Fisher Scientific, Rockford, IL, USA) using ChemiDoc XRS Gel Imager (Bio-Rad Laboratories, Inc., Hercules, CA, USA). Intensities of the protein bands were quantified using the ImageJ software $(\mathrm{NIH}, \mathrm{v} 1.47)$. In the quantitative analysis, ratios between various combinations of proteins and phosphorylated proteins were presented using ERK1 as a loading control by setting the control group value $(0 \mathrm{mg} / \mathrm{kg} / \mathrm{d}$ of PSH) at 1 .

\section{Statistical analyses}

The values are expressed as the means \pm standard error of the mean (SEM). Statistical analyses were performed using the 
SPSS software (IBM SPSS Statistics; version 19, Armonk, NY, USA). Shapiro-Wilk test and Levene's test were used to test all variables for normal distribution and homogeneity of variances, respectively. A one-way ANOVA followed by the post hoc test was used to compare changes in infarct size and western blot analysis. The statistical significance was set as $P<0.05$.

\section{RESULTS}

PSH supplementation reduces infarct size

Using a rat $\mathrm{I} / \mathrm{R}$ model, we first undertook to determine whether PSH supplementation protects against myocardial injury at the postocclusion steps. Our previous study showed that supplementation with $5 \mathrm{mg} / \mathrm{kg} / \mathrm{d}$ of $A X$ was enough to exert efficacies [26]. As $10 \mathrm{mg} / \mathrm{kg} / \mathrm{d}$ of PSH is equivalent to 5 $\mathrm{mg} / \mathrm{kg} / \mathrm{d}$ of $\mathrm{AX}$, considering that PSH contains approximately $50 \% A X$, we tested various dosages of PSH supplementation, including $10 \mathrm{mg} / \mathrm{kg} / \mathrm{d}$ of PSH (1, 10, or $100 \mathrm{mg} / \mathrm{kg} / \mathrm{d})$, to find a minimum efficacious dosage. The rats were fed diets supplemented with PSH for $3 \mathrm{~d}$, after which they were subjected to $30 \mathrm{~min}$ ischemia through ligation of LAD, followed by $3 \mathrm{~h}$ reperfusion through release of the ligation. Finally, the heart was harvested, cut into four slices, and stained with TTC (Fig. 2A). AAR, IA, and BZA were defined as the area without infiltration of Evans blue dye, the area without TTC stain, and the area equivalent to (AAR-IA), respectively (Fig. 2A). To determine whether PSH supplementation reduces myocardial injury, we assessed the infarct size (IS, the ratio of IA to AAR), which represents degree of cell death in the AAR. We observed that supplementation with 10 or $100 \mathrm{mg} / \mathrm{kg} / \mathrm{d}$ significantly reduces the IS, compared with the control group (39.8 \pm 3.3 and $38.3 \pm 4.9 \%$, respectively, versus $55.1 \pm 3.5 \% ; P<0.05$ ) (Fig. $2 \mathrm{~B}$ ). To confirm reproducibility of the experiments, we next assessed the risk size (RS, the ratio of AAR to LVA). The RS of PSH-treated groups was not significantly different from that of the control groups $(P>0.05)$, indicating that the surgical procedure to generate I/R injury was reliable (Fig. $2 \mathrm{~B}$ ). Taken together, these results indicate that PSH intake over certain dosages protects
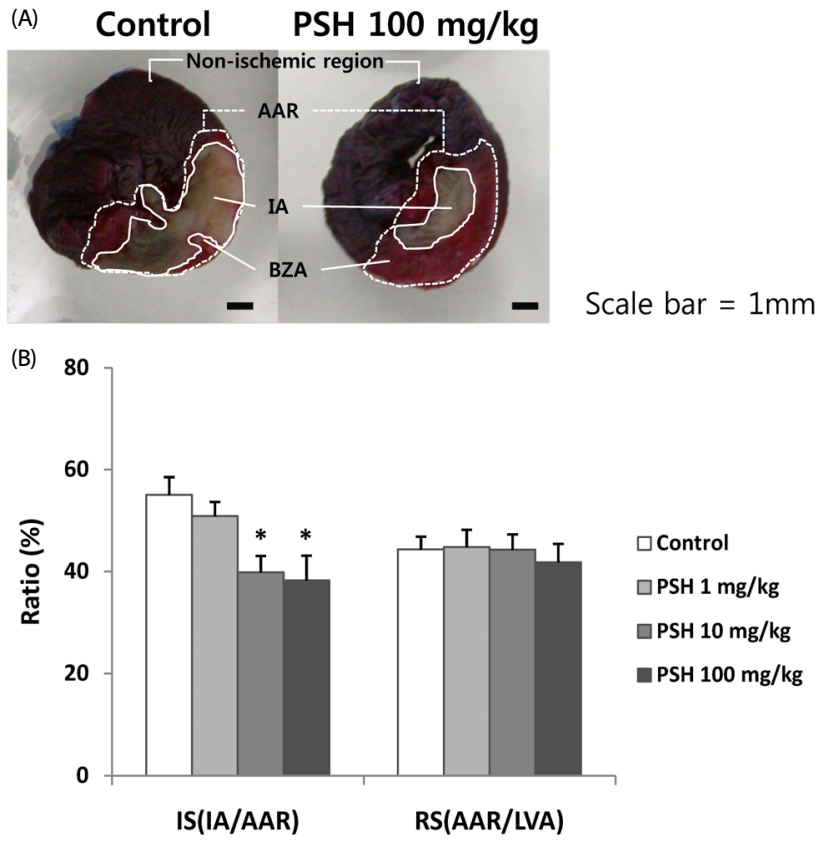

Fig. 2. Effect of PSH supplementation on infarct size. Rats underwent 30 min ischemia through ligation of $L A D$, followed by $3 \mathrm{~h}$ reperfusion through release of the ligation. (A) Evans blue dye was infused into the heart after LAD was re-ligated. The hear was harvested and cut into four slices. The slices were stained with TTC. AAR, IA, and BZA were determined as the area without infiltration of Evans blue dye, the area without TTC stain, and the area equivalent to (AAR-IA), respectively. (B) IS, the ratio of IA to AAR, and RS, the ratio of AAR to LVA, are presented. In the PSH-treated groups, PSH (1, 10 , or $100 \mathrm{mg} / \mathrm{kg} / \mathrm{d}$ ) supplements were fed for 3 days prior to LAD ligation. In the control group, no PSH was administered prior to LAD ligation. The number of rats used in the control and PSH-treated (1, 10, or $100 \mathrm{mg} / \mathrm{kg} / \mathrm{d})$ groups were 6 per group. Values are expressed as means \pm SEM. ${ }^{*} P<0.05$, when compared to control group. $\mathrm{PSH}$, psyllium seed husk; LAD, left anterior descending coronary artery; TTC, 2,3,5- triphenyltetrazolium chloride; AAR, area at risk; IA, infarct area; BZA, border zone area; IS, infarct size; RS, risk size; LVA; left ventricular area.

against $\mathrm{I} / \mathrm{R}$ injury during the postocclusion steps.

PSH supplementation reduces apoptosis

As AX supplementation reduces myocardial injury by inhibiting
(A)

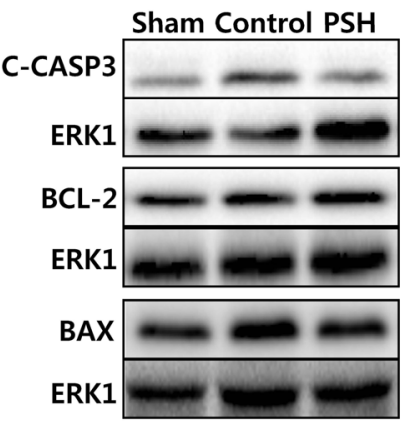

(B)

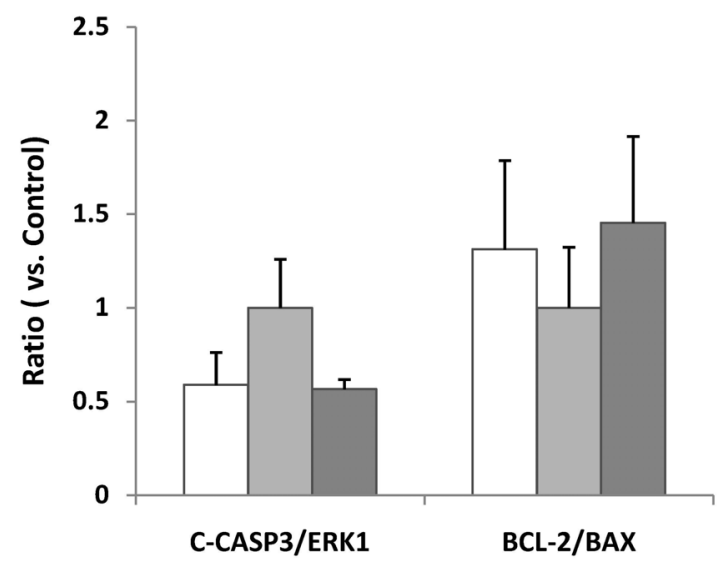

$\square$ Sham

$\square$ Control

$\square$ PSH $100 \mathrm{mg} / \mathrm{kg}$

Fig. 3. Effect of PSH supplementation on the formation of caspase-3 (CASP3) and expression of BCL-2 and BAX. (A) Western blots of CASP3 (cleaved caspase-3 generated from procaspase-3), BCL-2, and BAX in the AAR. Protein levels were measured by Western blotting for the sham, control, and PSH-treated (100 mg/kg/d) groups. ERK1 was used as the loading control. (B) Quantitative analysis of CASP3 (cleaved caspase-3), BCL-2, and BAX. C-CASP3 (CASP3)/ERK1 and BCL-2/BAX ratios are presented. The ratios were calculated by setting the control group value (0 mg/kg/d of PSH) at 1. The number of rats used in the sham, control and PSH-treated (100 mg/kg/d) groups were 6 per group. Values are expressed as means \pm SEM. CASP3, caspase-3; C-CASP3, cleaved caspase-3; PSH, psyllium seed husk; AAR, area at risk. 
apoptosis [26], we investigated whether PSH supplementation also protects against myocardial injury by inhibiting apoptosis, using CASP3 as a biomarker [30]. Supplementation with PSH (100 mg/kg/d) tends to reduce levels of CASP3 (cleaved CASP3 generated from procaspase-3) as compared with the control group, suggesting that PSH supplementation results in decreased apoptosis (Fig. 3A and 3B). To support the conclusion, we investigated whether $\mathrm{PSH}$ supplementation modulated the expression of $\mathrm{BCL}-2$ and $\mathrm{BAX}$, which are located upstream of CASP3 in the apoptotic cascade [31]. We observed that PSH supplementation tended to increase the ratio of $B C L-2$ with anti-apoptotic activities, and BAX with pro-apoptotic activities (BCL-2/BAX) as compared to the control group, suggesting that PSH supplementation contributes towards inhibiting apoptosis (Fig. 3B) $[31,32]$. Taken together, our results indicate that PSH intake protects against myocardial I/R injury by inhibiting the
(A)

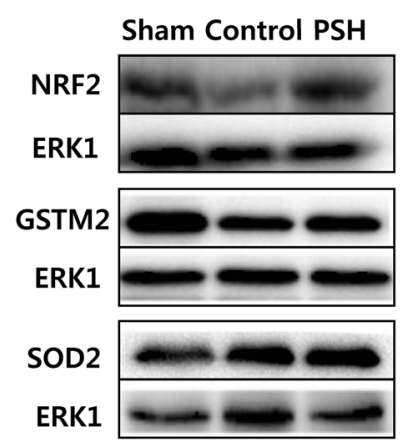

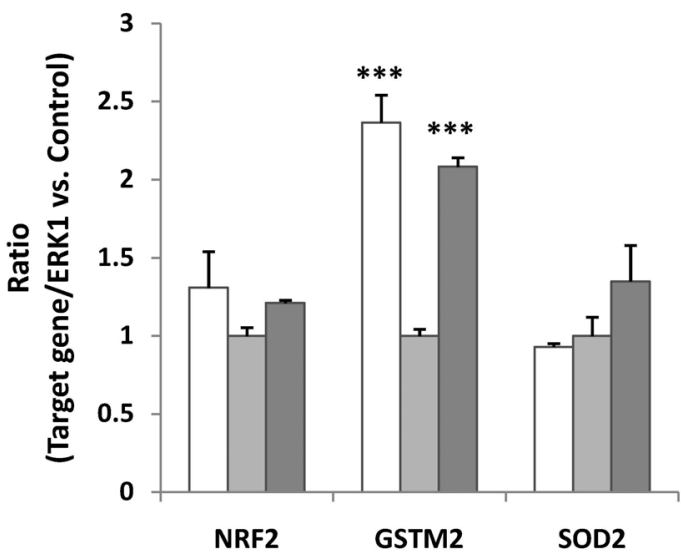

$\square$ Sham

$\square$ Control

$\square$ PSH $100 \mathrm{mg} / \mathrm{kg}$

Fig. 4. Effect of PSH supplementation on the expression of NRF2, GSTM2, and SOD2. Western blots of NRF2, GSTM2, and SOD2 in the AAR are presented. (A) Protein levels were measured by Western blotting for the sham, control, and PSH-treated (100 mg/kg/d) groups. ERK1 was used as a loading control. (B) Quantitative analysis of NRF2, GSTM2, and SOD2: NRF2/ERK1, GSTM2/ERK1, and SOD2/ERK1 ratios are presented. The ratios were calculated by setting the control group value (0 mg/kg/d of PSH) at 1 . The number of rats used in the sham, control and PSH-treated $(100 \mathrm{mg} / \mathrm{kg} / \mathrm{d})$ groups were 6 per group. Values are expressed as means $+\mathrm{SEM}$, ${ }^{* * *} P<0.001$, and * $P<0.05$ vs. control group. PSH, psyllium seed husk; NRF2, nuclear factor erythroid 2-related factor 2; GSTM2, glutathione S-transferase mu 2; SOD2, superoxide dismutase 2; AAR, area at risk.

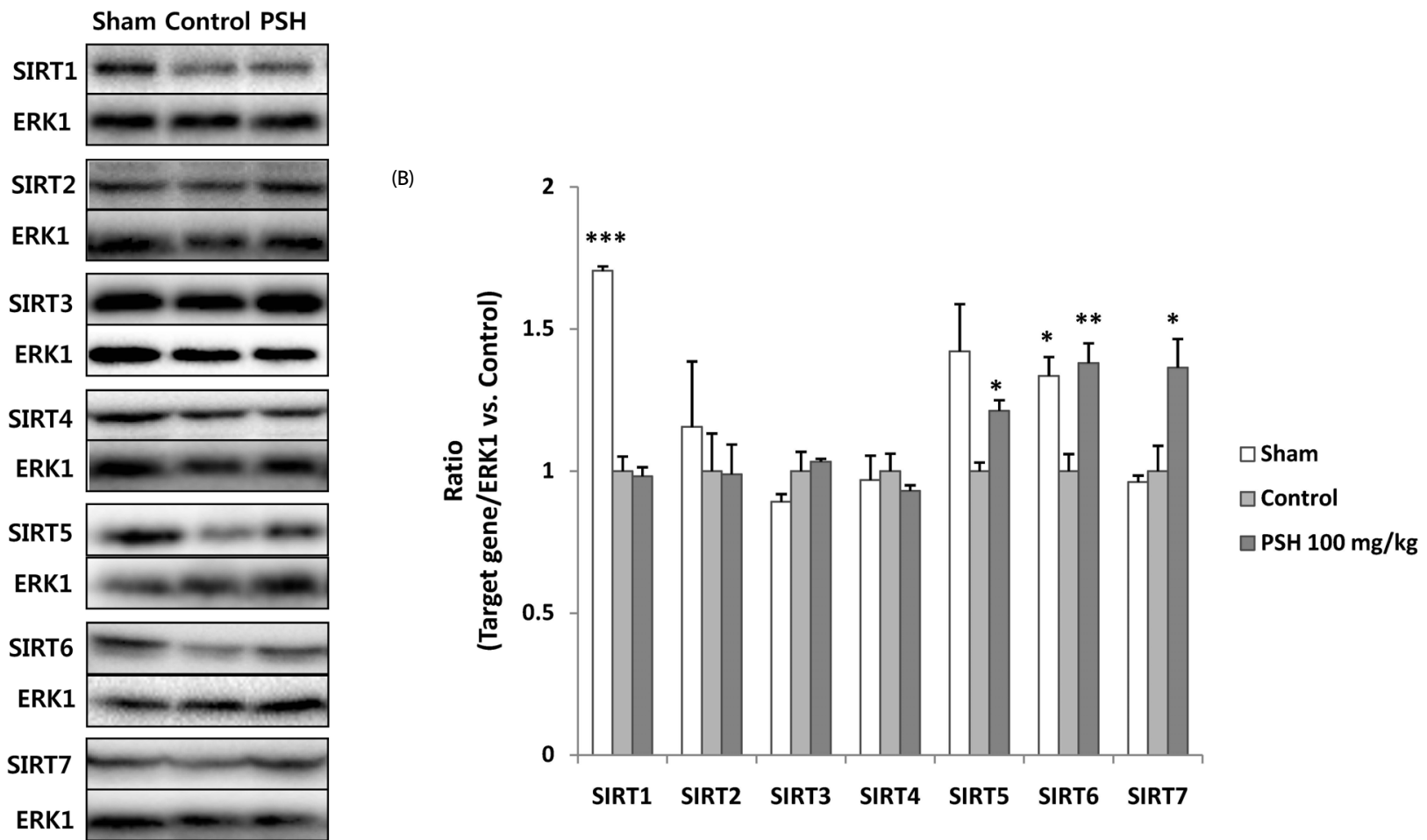

Fig. 5. Effect of PSH supplementation on the expression of SIRT (1-7). Western blots of SIRT (1-7) in the AAR are presented. (A) Protein levels were measured by Western blotting for the sham, control, and PSH-treated (100 mg/kg/d) groups. ERK1 was used as the loading control. (B) Quantitative analysis of SIRT (1-7): SIRT (1-7)/ERK1 ratios are presented. The ratios were calculated by setting the control group value $(0 \mathrm{mg} / \mathrm{kg} / \mathrm{d}$ of PSH) at 1 . The number of rats used in the sham, control and PSH-treated (100 mg/kg/d) groups were 6 per group. Values are expressed as means \pm SEM. ${ }^{\star \star} P<0.01$ and ${ }^{\star} P<0.05$ vs. control group. PSH, psyllium seed husk; SIRT, sirtuins; AAR, area at risk. 
(A)

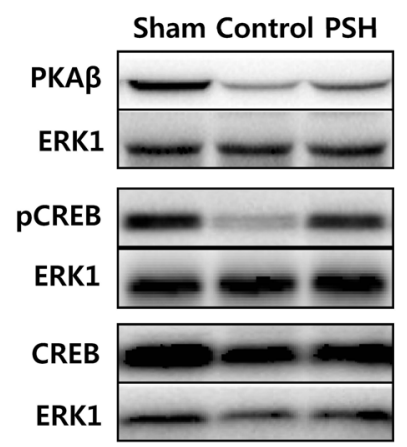

(B)

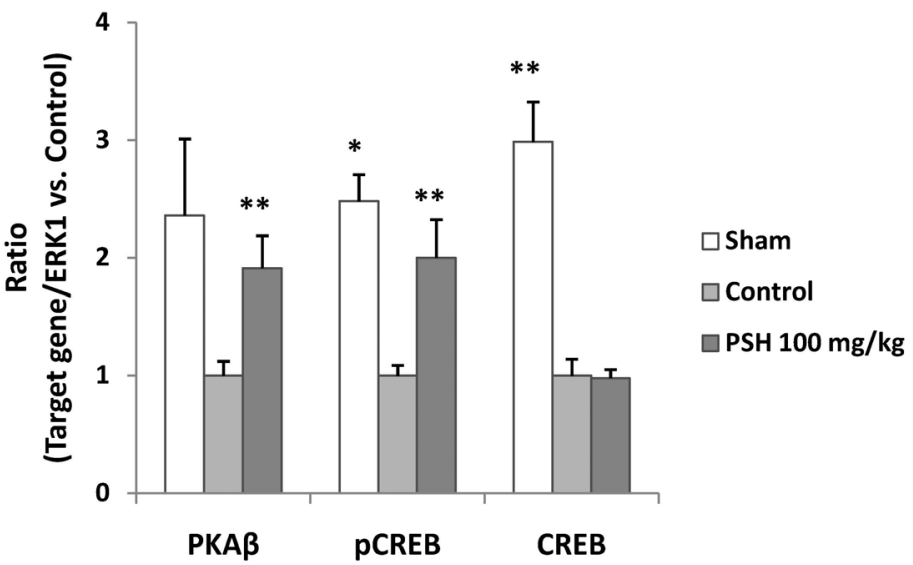

Fig. 6. Effect of PSH supplementation on the expression of PKA $\beta$, pCREB, and CREB. Western blots of PKAB, pCREB, and CREB in the AAR are presented. (A) Protein levels were measured by Western blotting for the sham, control, and PSH-treated (100 mg/kg/d) groups. ERK1 was used as the loading control, (B) Quantitative analysis of PKA $3 / E R K 1$, pCREB/ERK1, and CREB/ERK1 ratios are presented. The ratios were calculated by setting the control group value $(0 \mathrm{mg} / \mathrm{kg} / \mathrm{d}$ of PSH) at 1 . The number of rats used in the sham, control and PSH-treated $(100 \mathrm{mg} / \mathrm{kg} / \mathrm{d})$ groups were 6 per group. Values are expressed as means $\pm \mathrm{SEM}$. ${ }^{\star \star} P<0.01$ and ${ }^{\star} P<0.05$ vs. control group. PSH, psyllium seed husk; PKA ${ }^{\prime}$, protein kinase $A B$; pCREB, phosphorylated CAMP response element-binding protein; AAR, area at risk.

apoptotic cascades.

PSH supplementation upregulates expression of antioxidant enzymes through NRF2

We next investigated whether PSH supplementation upregulates the levels of GSTM2. PSH supplementation significantly upregulated GSTM2 expression compared with the control group (Fig. $4 \mathrm{~A}$ and $4 \mathrm{~B}$ ). In addition, PSH supplementation tended to upregulate the SOD2 expression, which is responsible for the removal of $\mathrm{O}_{2}^{-}$in the mitochondria. These results indicate that PSH supplementation eliminates ROS through upregulation of antioxidant enzymes. As both GSTM2 and SOD2 are target genes of NRF2 transcription factor [20,21], we investigated whether PSH supplementation upregulates NRF2 expression, and observed significant increase in NRF2 expression compared with the control (Fig. 4B). Taken together, our results indicate that PSH supplementation promotes the NRF2 expression, which contributes to upregulating gene expression of antioxidant enzymes.

\section{PSH supplementation upregulates expression of sirtuins}

The effect of PSH supplementation was investigated on the modulation of gene expression of sirtuins (Fig. 5A). Of the known sirtuins (1-7), SIRT5 (a mitochondrial sirtuin), and SIRT6 and SIRT7 (nuclear sirtuins) [22] were significantly upregulated by PSH supplementation (Fig. $5 \mathrm{~B}$ ). These results suggest that PSH supplementation upregulates some sirtuin genes, which might contribute to upregulating the NRF2 expression.

PSH supplementation promotes phosphorylation of CREB and upregulates expression of protein kinase $A(P K A)$

Finally, we investigated whether PSH supplementation increases the pCREB level (Fig. 6A). Indeed, PSH supplementation resulted in significant increases in the $\mathrm{PCREB}$ level compared to the control, although CREB levels remained unaffected (Fig. 6B). Moreover, as phosphorylation of CREB is mediated by PKA [33], we investigated whether $\mathrm{PSH}$ supplementation modulates the
PKA expression. PSH supplementation significantly increases PKA $\beta$, a catalytic subunit of PKA, compared to the control (Fig. $6 \mathrm{~B})$. Taken together, the results indicate that PSH supplementation upregulates PKA expression, which contributes to enhancing the phosphorylation of CREB.

\section{DISCUSSION}

In this study, we determined that PSH supplementation reduces myocardial $\mathrm{l} / \mathrm{R}$ injury by inhibiting the apoptotic cascades through reduction of CASP3 generation and increase of $\mathrm{BCL}-2 / \mathrm{BAX}$ ratio in a rat $\mathrm{I} / \mathrm{R}$ model. Inhibition of apoptosis might be mediated by from upregulation of PKA expression to reduction of CASP3 generation (Fig. 7). In the cascades, PSH supplementation first upregulates the PKA expression, which contributes to the enhancement of CREB phosphorylation to generate $\mathrm{PCREB}$. This theory is supported by previous studies which have reported that higher levels of PKA [24] and PCREB [23], and activation of PKA-CREB axis [34] are associated with myocardial protection against $\mathrm{I} / \mathrm{R}$ injury by inhibiting apoptosis through reduction of CASP3 generation and increase of BCL-2/BAX ratio. Therefore, our studies indicate that PSH supplementation contributes to myocardial protection by upregulation of PKA expression and enhancement of CREB phosphorylation. Of the 7 mammalian sirtuins (1-7), PSH supplementation upregulates the expression of SIRT5, SIRT6, and SIRT7. These findings indicate that $\mathrm{PSH}$ enhances the $\mathrm{NAD}^{+}$-dependent deacetylation of proteins including histones, in both mitochondria and nuclei, since SIRT5 is located in the mitochondria, and SIRT6 and SIRT7 are located in the nuclei [22]. Furthermore, it has been reported that higher levels of SIRT6 protect against myocardial I/R injury by inhibiting apoptosis through reduction of ROS generation, due to the upregulated antioxidant enzymes such as SOD2 [35]. In addition, higher levels of SIRT7 contribute to enhancing wound healing through increased scar formation after MI [36]. Finally, higher levels of SIRT5 protect the cardiomyocytes from oxidative stress by inhibiting apoptosis [37]. 


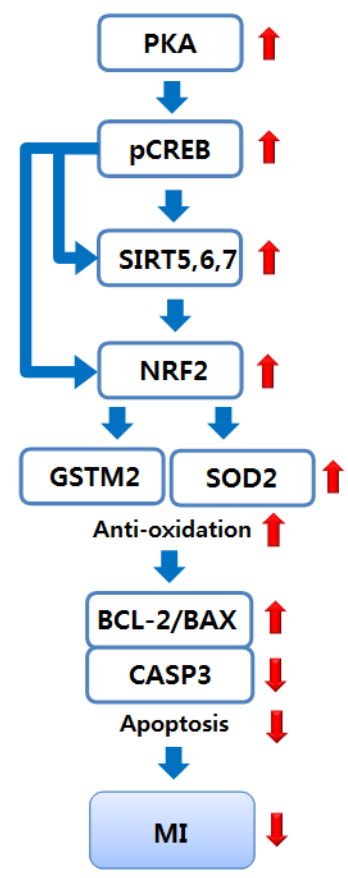

Fig. 7. A proposed, underlying mechanism for the myocardial protection through PSH supplementation. PSH supplementation protects against myocardial I/R injury through upregulation of PKA expression, CREB phosphorylation, sirtuin expression, NRF2 expression, expression of antioxidant enzymes such as GSTM2 and SOD2, and subsequent reduction of ROS toxicity, followed by reduction of apoptosis through increase of $B C L-2 / B A X$ ratio and subsequent reduction of CASP3 generation. PKA, protein kinase A; pCREB, phosphorylated cAMP response element-binding protein; SIRT5, 6, 7, sirtuins 5, 6, 7; NRF2, nuclear factor erythroid 2-related factor 2; GSTM2, glutathione S-transferase mu 2; SOD2, superoxide dismutase 2; CASP3, caspase-3; Ml, myocardial infarction; PSH, psyllium seed husk; I/R, ischemia/reperfusion; ROS, reactive oxygen species.

Taken together, PSH supplementation upregulates expression of some sirtuins such as SIRT5, SIRT6, and SIRT7, which contributes to protecting against myocardial I/R injury through elimination of ROS and subsequent enhancement of wound healing. Upregulation of the sirtuins through PSH supplementation might be mediated by binding of pCREB to the binding site CRE located on promoters of the sirtuins (SIRT6 gene promoter has putative pCREB binding site) [38] (Fig. 7). PSH supplementation also upregulates the expression of NRF2 and its target genes such as GSTM2 and SOD2 [20,21]. In our previous study, we showed that supplementation with wheat extract (WE) containing $\mathrm{AX}$ as an active component reduces the MDA generation and upregulates GSTM2 [26]. SOD2 is involved in removing $\mathrm{O}_{2}^{-}$in the mitochondria through conversion of $\mathrm{O}_{2}^{-}$ into $\mathrm{H}_{2} \mathrm{O}_{2}$ [18]. On the other hand, GSTM2 is involved in detoxification of HNE and MDA, the peroxidative byproducts of PUFA attributed to ROS, through conjugation of the byproducts with glutathione [19]. In addition, NRF2 expression is regulated by SIRT6 $[39,40]$ through deacetylation of histones [41]. The NRF2 gene promoter also has the pCREB binding site, implicating that CREB is also involved in the upregulation of NRF2 gene expression [42]. Taken together, these findings suggest that PSH supplementation upregulates NRF2 expression by $\mathrm{PCREB}$ and the sirtuins, which in turn results in upregulation of antioxidant enzymes and subsequent elimination of ROS toxicity (Fig. 7). Based on these results, we propose that PSH supplementation protects against myocardial I/R injury through upregulation of PKA expression, CREB phosphorylation, sirtuin expression, NRF2 expression, antioxidant enzymes expression, and subsequent reduction of ROS toxicity followed by reduction of apoptosis (Fig. 7).

The findings from this study indicating that PSH supplementation protects against myocardial I/R injury support our hypothesis that consumption of any foods containing high level of $\mathrm{AX}$ and/or arabinose (such as PSH) can be effective in protecting against myocardial injury. In this study, supplementation with PSH containing approximately $65 \%$ AX and $20 \%$ arabinose [28] showed efficacies at a dosage of $10 \mathrm{mg} / \mathrm{kg} / \mathrm{d}$. Previously, we showed that supplementation with AX containing approximately $40 \%$ arabinose exerts efficacies at a dosage of $5 \mathrm{mg} / \mathrm{kg} / \mathrm{d}$ [26]. We have also reported that supplementation with apple pectin containing approximately $1.5 \%$ arabinose shows efficacy at a dosage of $100 \mathrm{mg} / \mathrm{kg} / \mathrm{d}$ [10]. Therefore, this suggests that consumption of lesser amount of foods containing higher levels of $\mathrm{AX}$ or arabinose are required to exhibit their efficacies. An underlying mechanism by which PSH consumption protects against myocardial injury through arabinose can be presented, as previously described [26]. Briefly, once PSH is ingested, AX present in PSH is hydrolyzed to arabinose and xylose by microbiota inhabiting the large intestine. In turn, the arabinose generated is absorbed into the body and protects the heart against ischemic injury.

Regardless of underlying mechanisms, the findings from this study reveal that short-term PSH intake protects against myocardial I/R injury even after coronary arteries are occluded, indicating that PSH intake can prevent MI by blocking the postocclusion steps (Fig. 1). It has already been shown that long-term PSH consumption prevents $\mathrm{Ml}$ by blocking the preocclusion steps, because PSH consumption was effective in reducing risk factors for coronary ischemic disease such as hyperlipidemia [11,12], diabetes [14], and hypertension [15], which promote the formation of atherosclerotic plaques responsible for narrowing the coronary arteries (Fig. 1). Overall, we conclude that PSH consumption prevents $\mathrm{MI}$ by inhibiting both the preocclusion and postocclusion steps.

Approximately $10 \mathrm{~g} / \mathrm{d}$ of PSH was administered in various clinical trials to assess the efficacies in reducing the risk factors $[11,14,15]$. Based on these findings, the US Food and Drug Administration (FDA) approved health claims of reducing cardiovascular disease for PSH at dosages of over $8 \mathrm{~g} / \mathrm{d}$ [43]. However, results from the current study indicate that PSH supplementation to the rats protected myocardial I/R injury at dosages over $10 \mathrm{mg} / \mathrm{kg} / \mathrm{d}$. The dosage is equivalent to $100 \mathrm{mg} / \mathrm{d}$ for a $60 \mathrm{~kg}$ person, based on calculations using a conversion factor provided by the FDA [44]. Thus, we postulate that the dosage required to show efficacies for blocking postocclusion steps is much smaller than that required for blocking preocclusion steps, which will therefore not cause any safety concerns for consuming PSH to reduce myocardial injury.

In conclusion, the results from our study reveal that PSH consumption reduces myocardial $\mathrm{I} / \mathrm{R}$ injury in rats by inhibiting the apoptotic cascades through modulation of gene expression of several genes located upstrem of apoptosis, at a safe dosage 
when converted to human beings. Our findings indicate that PSH consumption prevents Ml by blocking the postocclusion steps. We belive that PSH has the potential to be developed as a functional food for preventing $\mathrm{Ml}$, through blockade of both the preocclusion and postocclusion steps.

\section{CONFLICT OF INTEREST}

The authors declare no potential conflicts of interests.

\section{ORCID}

Sun Ha Lim: https://orcid.org/0000-0002-8371-4740

Jongwon Lee: https://orcid.org/0000-0001-9372-9850

\section{REFERENCES}

1. Lim SH, Lee J. Xyloglucan intake attenuates myocardial injury by inhibiting apoptosis and improving energy metabolism in a rat model of myocardial infarction. Nutr Res 2017;45:19-29.

2. Lim SH. Larch arabinogalactan attenuates myocardial injury by inhibiting apoptotic cascades in a rat model of ischemiareperfusion. J Med Food 2017;20:691-9.

3. Kim MY, Lim SH, Lee J. Intake of hot water-extracted apple protects against myocardial injury by inhibiting apoptosis in an ischemia/reperfusion rat model. Nutr Res 2014;34:951-60.

4. Lim SH, Kim MJ, Han MJ, Kim Y, Lee J. Prevention of ischemic diseases and cognitive disorders through wheat consumption. In: Duncan LT, editor. Advances in Health and Disease. Hauppauge (NY): Nova Science Publishers, Inc.; 2018. p.1-66.

5. Soler EP, Ruiz VC. Epidemiology and risk factors of cerebral ischemia and ischemic heart diseases: similarities and differences. Curr Cardiol Rev 2010;6:138-49.

6. Hajar R. Risk factors for coronary artery disease: historical perspectives. Heart Views 2017;18:109-14.

7. Hurtubise J, McLellan K, Durr K, Onasanya O, Nwabuko D, Ndisang JF. The different facets of dyslipidemia and hypertension in atherosclerosis. Curr Atheroscler Rep 2016;18:82.

8. Park KH, Park WJ. Endothelial dysfunction: clinical implications in cardiovascular disease and therapeutic approaches. J Korean Med Sci 2015;30:1213-25.

9. Maiolino G, Rossitto G, Caielli P, Bisogni V, Rossi GP, Calò LA. The role of oxidized low-density lipoproteins in atherosclerosis: the myths and the facts. Mediators Inflamm 2013;2013:714653.

10. Lim SH, Kim MY, Lee J. Apple pectin, a dietary fiber, ameliorates myocardial injury by inhibiting apoptosis in a rat model of ischemia/reperfusion. Nutr Res Pract 2014;8:391-7.

11. Wei ZH, Wang $H$, Chen XY, Wang BS, Rong ZX, Wang BS, Su BH, Chen HZ. Time- and dose-dependent effect of psyllium on serum lipids in mild-to-moderate hypercholesterolemia: a meta-analysis of controlled clinical trials. Eur J Clin Nutr 2009;63:821-7.

12. Ribas SA, Cunha DB, Sichieri R, Santana da Silva LC. Effects of psyllium on LDL-cholesterol concentrations in Brazilian children and adolescents: a randomised, placebo-controlled, parallel clinical trial. Br J Nutr 2015;113:134-41.

13. Xing LC, Santhi D, Shar AG, Saeed M, Arain MA, Shar AH, Bhutto ZA, Katar MU, Manzoor R, El-Hack ME, Alagawany M, Dhama K, Ling MC. Psyllium husk (Plantago ovata) as a potent hypocholesterolemic agent in animal, human and poultry. Int J Pharmacol 2017;13:690-7.

14. Gibb RD, McRorie JW Jr, Russell DA, Hasselblad V, D'Alessio DA Psyllium fiber improves glycemic control proportional to loss of glycemic control: a meta-analysis of data in euglycemic subjects, patients at risk of type 2 diabetes mellitus, and patients being treated for type 2 diabetes mellitus. Am J Clin Nutr 2015;102: 1604-14.

15. Khan K, Jovanovski E, Ho HV, Marques AC, Zurbau A, Mejia SB, Sievenpiper JL, Vuksan V. The effect of viscous soluble fiber on blood pressure: a systematic review and meta-analysis of randomized controlled trials. Nutr Metab Cardiovasc Dis 2018;28:3-13.

16. Obata K, Ikeda K, Yamasaki M, Yamori Y. Dietary fiber, psyllium, attenuates salt-accelerated hypertension in stroke-prone spontaneously hypertensive rats. J Hypertens 1998;16:1959-64.

17. Raedschelders K, Ansley DM, Chen DD. The cellular and molecular origin of reactive oxygen species generation during myocardial ischemia and reperfusion. Pharmacol Ther 2012;133:230-55.

18. Zhang $Y$, Martin SG. Redox proteins and radiotherapy. Clin Oncol ( $R$ Coll Radiol) 2014;26:289-300.

19. Ayala A, Muñoz MF, Argüelles S. Lipid peroxidation: production, metabolism, and signaling mechanisms of malondialdehyde and 4-hydroxy-2-nonenal. Oxid Med Cell Longev 2014;2014:360438.

20. Zhou S, Sun W, Zhang Z, Zheng Y. The role of Nrf2-mediated pathway in cardiac remodeling and heart failure. Oxid Med Cell Longev 2014;2014:260429.

21. Murphy KE, Park JJ. Can co-activation of Nrf2 and neurotrophic signaling pathway slow Alzheimer's disease? Int J Mol Sci 2017;18:1168

22. Matsushima S, Sadoshima J. The role of sirtuins in cardiac disease. Am J Physiol Heart Circ Physiol 2015;309:H1375-89.

23. Yu W, Xu M, Zhang T, Zhang Q, Zou C. Mst1 promotes cardiac ischemia-reperfusion injury by inhibiting the ERK-CREB pathway and repressing FUNDC1-mediated mitophagy. J Physiol Sci 2019;69: 113-27.

24. Zhang Y, Wang XL, Zhao J, Wang YJ, Lau WB, Yuan YX, Gao EH, Koch WJ, Ma XL. Adiponectin inhibits oxidative/nitrative stress during myocardial ischemia and reperfusion via PKA signaling. Am J Physiol Endocrinol Metab 2013;305:E1436-43.

25. Arai AE. Healing after myocardial infarction: a loosely defined process. JACC Cardiovasc Imaging 2015;8:680-3.

26. Lim SH, Kim Y, Yun KN, Kim JY, Jang JH, Han MJ, Lee J. Plant-based foods containing cell wall polysaccharides rich in specific active monosaccharides protect against myocardial injury in rat myocardial infarction models. Sci Rep 2016;6:38728.

27. Van Craeyveld V, Delcour JA, Courtin CM. Ball milling improves extractability and affects molecular properties of psyllium (Plantago ovata Forsk) seed husk arabinoxylan. J Agric Food Chem 2008;56: 11306-11.

28. Lim SH, Kim MJ, Lee J. Intake of psyllium seed husk reduces white matter damage in a rat model of chronic hypoperfusion. Nutr Res 2019; https://doi.org/10.1016/j.nutres.2019.04.002

29. Lim SH, Lee J. Protection of the brain through supplementation with larch arabinogalactan in a rat model of vascular dementia. Nutr Res Pract 2017;11:381-7.

30. Porter AG, Jänicke RU. Emerging roles of caspase-3 in apoptosis Cell Death Differ 1999;6:99-104.

31. Youle RJ, Strasser A. The BCL-2 protein family: opposing activities that mediate cell death. Nat Rev Mol Cell Biol 2008;9:47-59. 
32. Edlich F. BCL-2 proteins and apoptosis: recent insights and unknowns. Biochem Biophys Res Commun 2018;500:26-34.

33. Sands WA, Palmer TM. Regulating gene transcription in response to cyclic AMP elevation. Cell Signal 2008;20:460-6.

34. Zhao D, Feng $P$, Sun $Y$, Qin $Z$, Zhang Z, Tan $Y$, Gao E, Lau WB, Ma X, Yang J, Yu S, Xu X, Yi D, Yi W. Cardiac-derived CTRP9 protects against myocardial ischemia/reperfusion injury via calreticulindependent inhibition of apoptosis. Cell Death Dis 2018;9:723.

35. Wang XX, Wang XL, Tong MM, Gan L, Chen H, Wu SS, Chen JX, Li RL, Wu Y, Zhang HY, Zhu Y, Li YX, He JH, Wang M, Jiang W. SIRT6 protects cardiomyocytes against ischemia/reperfusion injury by augmenting FoxO3a-dependent antioxidant defense mechanisms. Basic Res Cardiol 2016;111:13.

36. Araki $S$, Izumiya $Y$, Rokutanda $T$, lanni $A$, Hanatani $S$, Kimura $Y$, Onoue $\mathrm{Y}$, Senokuchi T, Yoshizawa T, Yasuda O, Koitabashi N, Kurabayashi M, Braun T, Bober E, Yamagata K, Ogawa H. Sirt7 contributes to myocardial tissue repair by maintaining transforming growth factor- $\beta$ signaling pathway. Circulation 2015;132:1081-93.

37. Liu B, Che W, Zheng C, Liu W, Wen J, Fu H, Tang K, Zhang J, Xu Y. SIRT5: a safeguard against oxidative stress-induced apoptosis in cardiomyocytes. Cell Physiol Biochem 2013;32:1050-9.

38. Fishilevich S, Nudel R, Rappaport N, Hadar R, Plaschkes I, Iny Stein $T$, Rosen N, Kohn A, Twik M, Safran M, Lancet D, Cohen D. GeneHancer: genome-wide integration of enhancers and target genes in GeneCards. Database (Oxford) 2017;2017:1

39. Pan H, Guan D, Liu X, Li J, Wang L, Wu J, Zhou J, Zhang W, Ren R, Zhang W, Li Y, Yang J, Hao Y, Yuan T, Yuan G, Wang H, Ju Z, Mao Z, Li J, Qu J, Tang F, Liu GH. SIRT6 safeguards human mesenchymal stem cells from oxidative stress by coactivating NRF2. Cell Res 2016;26:190-205

40. Zhang W, Wei R, Zhang L, Tan Y, Qian C. Sirtuin 6 protects the brain from cerebral ischemia/reperfusion injury through NRF2 activation. Neuroscience 2017;366:95-104.

41. Liao CY, Kennedy BK. SIRT6, oxidative stress, and aging. Cell Res 2016;26:143-4.

42. Impey S, McCorkle SR, Cha-Molstad H, Dwyer JM, Yochum GS, Boss JM, McWeeney S, Dunn JJ, Mandel G, Goodman RH. Defining the CREB regulon: a genome-wide analysis of transcription factor regulatory regions. Cell 2004;119:1041-54.

43. Jenkins $D J$, Kendall CW, Vuksan V, Vidgen $E$, Parker T, Faulkner $D_{t}$ Mehling CC, Garsetti M, Testolin G, Cunnane SC, Ryan MA, Corey PN. Soluble fiber intake at a dose approved by the US Food and Drug Administration for a claim of health benefits: serum lipid risk factors for cardiovascular disease assessed in a randomized controlled crossover trial. Am J Clin Nutr 2002;75:834-9.

44. Nair $A B$, Jacob $S$. A simple practice guide for dose conversion between animals and human. J Basic Clin Pharm 2016;7:27-31. 